Vol. 22, 2020/e09

\title{
Caracterización de la comunicación interpersonal en el proceso enseñanza-aprendizaje
}

\section{Characterization of Interpersonal Communication in the Teaching-Learning Process}

\author{
Marivel Jurado Ronquillo (1) http://orcid.org/0000-0001-9455-1441 \\ Raidell Avello Martínez (2) http://orcid.org/0000-0001-7200-632X \\ Gisela Bravo López (2) http://orcid.org/0000-0001-6818-8466 \\ (1) Universidad de Guayaquil \\ (2) Universidad de Cienfuegos \\ (Recibido: 28 de febrero de 2018; Aceptado para su publicación: 2 de mayo de 2018)
}

Cómo citar: Jurado, M., Avello, R. y Bravo, G. (2020). Caracterización de la comunicación interpersonal en el proceso enseñanzaaprendizaje. Revista Electrónica de Investigación Educativa, 22, e09, 1-11. https://doi.org/10.24320/redie.2020.22.e09.2284

\section{Resumen}

El análisis de las concepciones teóricas de la comunicación permite identificar la importancia que se le concede a ésta en la formación del hombre; la comunicación debe ser estudiada como objetivo y contenido del currículo o como un saber transversal. El objetivo de esta investigación es caracterizar la comunicación interpersonal en el proceso enseñanza-aprendizaje en la Licenciatura en Educación de la Universidad de Guayaquil (Ecuador). Los instrumentos utilizados fueron la observación y el cuestionario; la muestra estuvo conformada por 62 profesores y 355 estudiantes. Como principales resultados se aprecian limitaciones en la proyección didáctica de la comunicación interpersonal en el proceso enseñanza-aprendizaje, lo cual se evidencia en la falta de diálogo, carencia de actividades grupales durante las clases e insuficiencia en la personalización del proceso, entre otras.

Palabras clave: Comunicación interpersonal, proceso enseñanza-aprendizaje, educación superior.

\section{Abstract}

An analysis of theoretical understandings of communciation makes it possible to identify its importance in personal development; communication should be studied as a curricular objective and as curricular content, or as cross-disciplinary knowledge. The aim of this research is to characterize interpersonal communication in the teaching-learning process in the bachelor's degree in education in the University of Guayaquil (Universidad de Guayaquil) in Ecuador. The instruments used were observation and questionnaires. The sample was made up of 62 teachers and 355 students. The main findings include limitations in the didactic projection of interpersonal communication in the teaching-learning process, which is shown in the lack of dialogue, a lack of group activities during classes, and insufficient personalization of the process, among other aspects. 


\section{Introducción}

Los estudios acerca del proceso de enseñanza-aprendizaje indican la necesidad de perfeccionar la intervención del profesor en calidad de mediador en éste. Aseguran que desde su gestión el profesor debe propiciar actividades que permitan al estudiante en formación aprender a comunicarse (Johnson et al., 2017), ajustado a las exigencias de la actividad profesional para la que se forman; permitiéndoles compartir con su grupo, lo que resulta imprescindible para poder comprender, expresarse e interrelacionarse y mejorar la realidad y el contexto en que viven y trabajan (Amayuela, 2005; Contreras, 1990).

El análisis de las concepciones teóricas de la comunicación permiten identificar la importancia que se le concede a ésta en la formación del hombre, idea que es fundamentada desde Elkonin (1982) y Leontiev (1985), quienes conciben la actividad como una condición de la comunicación, hasta Lomov (1987), quien considera que en la comunicación se descubre el mundo subjetivo de un hombre por otro hombre y que en ella se manifiestan las cualidades y propiedades que lo caracterizan como personalidad (Hargie, 2016; Tejeda, 2010; Turró, 2017).

Desde estos referentes, la comunicación debe ser estudiada como objetivo y contenido del currículo, o como un saber transversal (Berger, 2014). Sin embargo, en las carreras de Educación se le atribuye un carácter especializado, definida como pedagógica, educativa o didáctica para referirse a las exigencias y requisitos de la actividad profesional y el contexto en que se realiza (Bravo, 2004; Portela, 2015).

A nivel internacional y nacional, con independencia de la posición asumida por diferentes autores (Alpizar, 2017; Johnson et al., 2017; Sánchez, 2000), en sus planteamientos se revela la necesidad de la comunicación interpersonal en el proceso enseñanza-aprendizaje, y por la intención de sus propuestas se convierten en referentes teórico-metodológicos y antecedentes valiosos para toda investigación en el tema. Sin embargo, desde el punto de vista metodológico no dejan planteadas, de forma explícita, la manera de relacionar ambos procesos.

Los autores consultados insisten en declarar que la comunicación ha sido tratada como competencia o habilidad necesaria para que el profesor desarrolle su función didáctica (Punyanunt-Carter y Arias, 2017); no obstante, son insuficientes las actividades que se realizan desde el proceso de formación para obtener los conocimientos y las habilidades necesarias, con el propósito de convertirse en verdaderos innovadores de sus prácticas pedagógicas futuras y puedan utilizar la comunicación en el grupo clase, potenciar un proceso de enseñanza-aprendizaje basado en el diálogo y la reflexión (Heredero, y Garrido, 2017; Sánchez y Boronat, 2014).

Los resultados del análisis de documentos -y las encuestas aplicadas con el fin de indagar en esta situacióncoinciden en que si bien el profesor es un comunicador por excelencia, los estudiantes que se forman en las carreras de educación reciben una preparación insuficiente que limita la capacidad para aprovechar las potencialidades de la comunicación interpersonal en su labor e insisten en que las propuestas orientadas a tratar de resolver esta situación no rebasan, en su generalidad, la introducción de asignaturas que abordan, sin que constituya un rasgo distintivo del proceso de enseñanza-aprendizaje en la formación del estudiante de Educación.

De igual manera, al valorar el tema con estudiantes y egresados de la Licenciatura en Educación de la Universidad de Guayaquil, profesores y directivos de las universidades en la provincia de Guayas, Loja, Pichincha y Manabí (Ecuador), se constató que sus criterios coinciden con investigaciones precedentes (Jurado, Bravo, y Muñoz, 2016; Jurado, Bravo y Avello, 2017), manifestando lo siguiente:

- Existe poco conocimiento de las estrategias orientadas a favorecer la participación de los estudiantes y el trabajo en grupo como espacio para potenciar la comunicación interpersonal.

- Reconocen que las actividades de enseñanza-aprendizaje se organizan de manera tal que ofrecen pocas oportunidades para ejercitar la comunicación interpersonal, dado por el modelo unidireccional que generalmente se sigue en estas instituciones. 
- No se utilizan con frecuencia las posibilidades del diálogo como una vía para que el profesor universitario se convierta en modelo de comunicación interpersonal, pues los métodos reproductivos son los más utilizados.

- Las clases ofrecen pocas oportunidades para que los estudiantes intercambien ideas, reflexiones y puntos de vista durante el desarrollo de las mismas.

- No se identifica el método como una oportunidad para ampliar los conocimientos y habilidades de la comunicación interpersonal.

Dicho lo anterior, es preciso superar la concepción de la comunicación interpersonal en el proceso de enseñanza-aprendizaje, aunque el tema se trabaja en las diversas asignaturas no se analiza la relación desde lo metodológico -entre la comunicación interpersonal y dicho proceso-, lo cual explica (desde el punto de vista práctico) que sea necesario identificar los métodos que pueden utilizarse, para lograr dicho propósito en los estudiantes de la Licenciatura en Educación.

Esta situación confirma la contradicción entre las limitadas concepciones acerca de la comunicación interpersonal en el proceso de enseñanza-aprendizaje y las demandas de formación del estudiante universitario. Por ello el objetivo de esta investigación es caracterizar la comunicación interpersonal en el proceso de enseñanza-aprendizaje en la Licenciatura en Educación de la Universidad de Guayaquil (Ecuador).

\section{Método}

La investigación se realizó en la Licenciatura en Educación de la Facultad de Filosofía Letras y Ciencias de la Educación de la Universidad de Guayaquil (Ecuador). Para su realización se tuvo en cuenta que el acceso al campo estuviera respaldado por las autoridades del centro y contara con la aceptación de profesores y estudiantes, por lo que se les informó la importancia del estudio y los objetivos.

Las indagaciones sobre de la situación que presenta en la práctica la formación de los estudiantes de la Licenciatura en Educación y la repercusión de la comunicación interpersonal en el proceso de enseñanzaaprendizaje fueron los aspectos clave para estructurar las ideas básicas presentadas en la introducción.

Para la elaboración de los instrumentos empleados en la caracterización del objeto de investigación se tuvo en cuenta: asegurar su objetividad, por lo que su construcción se presenta como un paso, en el cual el investigador se implica para ajustar y asegurar el rigor de los mismos al propósito planteado (Cohen et al., 2013). Los investigadores realizaron una prueba piloto como criterio de validez esencial de los cuestionarios, para los que se determinó el nivel de confiabilidad utilizando el coeficiente Alfa de Cronbach, los valores obtenidos fueron mayores de 0.75 -considerado adecuado para investigaciones del área de Ciencias Sociales (Hernández et al., 2014).

Es importante aclarar que los instrumentos contienen aspectos específicos y de relación entre ellos, de manera que permiten la interpretación cruzada de la información, entre los que se encuentran: cómo tiene lugar la comunicación interpersonal, cómo influye en el proceso de enseñanza-aprendizaje y cómo el profesor contribuye a la comunicación interpersonal.

A continuación se presenta una descripción de cada instrumento elaborado y aplicado para la caracterización de la comunicación interpersonal en el proceso de enseñanza- aprendizaje.

Observaciones en clases. La observación constituye un método fundamental en la caracterización de cualquier fenómeno psicopedagógico, permite la recopilación de la información primaria acerca del objeto de estudio, aportando una mayor objetividad cuando la información a obtener es susceptible de ser cuantificada. 
Tomando esta idea como punto de partida se diseñó la guía de observación para caracterizar la manera en que tiene lugar la comunicación interpersonal y cómo influye en el proceso de enseñanza-aprendizaje. La guía de observación elaborada, en su primera versión, contó con una escala analítica para el procesamiento y la interpretación de los datos, pero luego, a partir de las opiniones de los especialistas, se elaboró una segunda variante con una escala analítico-sintética que permitiera graduar los comportamientos con mayor ajuste.

La guía de observación se divide en tres apartados, que coinciden con las fases del proceso de enseñanza aprendizaje: I) Orientación, II) Ejecución y III) Evaluación, con 4, 4, y 5 elementos de observación cada una respectivamente. Además, incluye una sección final para observaciones adicionales y otros criterios del observador.

Se realizaron 57 observaciones en clases de todos los semestres a los profesores a tiempo completo y a medio tiempo, vinculados a la carrera por más de seis años y que imparten clases en varios semestres. El tiempo de cada observación fue de una hora de clase.

Otro de los métodos fue el autorreporte tipo inventario aplicado a los profesores, el cual consta de 44 ítems, y las posibilidades de respuestas son: $X$ (aquellas afirmaciones que considere están en correspondencia con las características de la comunicación que usted establece con sus estudiantes), 0 (aquellas con las cuales no esté de acuerdo) y ? (en caso de duda o indecisión), se parte de que la comunicación interpersonal que se desarrolla en el proceso de enseñanza-aprendizaje tiene un papel determinante en la formación de la personalidad de los sujetos, sobre todo por el intercambio cognoscitivo y afectivo que tiene lugar en éste. Derivado de lo anterior, se consideró necesario estudiar de manera autorreflexiva el comportamiento de esta relación a partir de las funciones del emisor y receptor.

Esta exploración tuvo como objetivo llegar a una caracterización general de la comunicación interpersonal a partir de algunos elementos específicos, que son esenciales en el proceso de enseñanza-aprendizaje, y otros que influyen en la comunicación interpersonal.

En la elaboración de este cuestionario, y los que se mencionan a continuación, se tuvieron en cuenta los siguientes aspectos:

- La formulación de los ítems en un lenguaje claro, breve y preciso.

- Elaboración de una adecuada cantidad de ítems, de modo que pueda ser respondido en un lapso prudente de tiempo.

- El balance equitativo entre la cantidad de ítems positivos y negativos.

- El ordenamiento aleatorio en términos de las áreas a explorar, con respecto a la direccionalidad positiva o negativa de los ítems.

En el cuestionario de opinión aplicado a los profesores, que cuenta con 9 ítems y posibilidades de respuesta: sí, no y por qué, se consideró combinar preguntas cerradas y abiertas, ofreciendo la posibilidad de que el participante argumentara sus opiniones. Este cuestionario tuvo como objetivo indagar cómo el profesor contribuye a la comunicación interpersonal en el proceso de enseñanza-aprendizaje

Para estudiar a profundidad las causas esenciales que propician una comunicación interpersonal que favorezca el proceso de enseñanza-aprendizaje en la formación de los estudiantes en la Licenciatura en Educación, fue necesario indagar en los niveles de satisfacción que poseen acerca de la manera en que despliegan la comunicación interpersonal en el proceso, por lo que se elaboró un cuestionario para estudiantes -con 34 ítems y posibilidades de respuestas similares al autorreporte aplicado a los profesores.

Se utilizó otro cuestionario para revelar la valoración que poseen los estudiantes acerca del tema y los aprendizajes que han logrado a través de éste. El cuestionario incluye, primero, una escala ordinal que permite conformar un rango de esa contribución, y completa la información con una ponderación de mayor o menor que evidencia el logro de los propósitos formativos en este sentido. 
El proceso de aplicación incluyó un muestreo intencional y se consideró su representatividad con respecto a la población sobre la base del número de estudiantes e impacto social de los procesos formativos, pues se previó tomar en consideración la posibilidad de mantener el trabajo con las muestras durante la validación de la propuesta. La tabla I describe las muestras y criterios de selección.

Tabla I. Distribución de la población y muestra para las encuestas a profesores y estudiantes

\begin{tabular}{l|c|l|c}
\hline Sujetos implicados & Población & \multicolumn{1}{|c|}{ Instrumentos } & Muestras \\
\hline Profesores & 154 & Observaciones, cuestionarios & 62 \\
Estudiantes & 5032 & Cuestionarios & 355 \\
\hline
\end{tabular}

\section{Resultados y discusión}

Antes de iniciar los autores revisaron los planes de estudio de varias universidades iberoamericanas, así como informes que incluyen la valoración del proceso de formación de los estudiantes en la Licenciatura en Educación, e identificó como regularidad que no se revela la relación necesaria de este conocimiento con la actividad profesional que realizarán una vez graduados.

En la revisión se constató que las propuestas curriculares están orientadas más a la especialización de contenidos de la comunicación que son trabajados en asignaturas, lo que limita su aplicación en el proceso de enseñanza-aprendizaje en que se forman los futuros educadores y que constituye una característica y exigencia del perfil de formación del licenciado en Educación.

\subsection{Observaciones}

Las observaciones se realizaron como invitado, acordando con el profesor que la participación del investigador no sería divulgada ni tomada en cuenta para la evaluación de las clases; ya que su propósito era la investigación de la práctica.

Cabe señalar que en la fase de orientación los profesores se limitaban a establecer preguntas como la vía fundamental para controlar o conocer cómo había sido estudiado lo orientado en el trabajo independiente y las dificultades que habían tenido para su realización. No se aprovecha este momento para escuchar diferentes criterios y posibilitar la participación de los estudiantes del grupo; en su mayoría los profesores visitados tratan de motivar la clase al comienzo de ésta, pero no mantienen la motivación durante el desarrollo de las actividades de enseñanza-aprendizaje, por lo que los estudiantes pierden el interés hacia el tema que se está trabajando y no participan activamente. La comunicación se da, fundamentalmente, entre el profesor y los estudiantes, limitándose entre los estudiantes.

En la fase de ejecución los profesores mantienen el papel rector del proceso, lo cual si bien facilita la utilización y construcción de los conocimientos, no se aprovechan las experiencias previas que tienen los estudiantes y durante el desarrollo de las clases prima la interacción profesor-estudiante, sólo 18 profesores organizan sus clases atendiendo este aspecto y potencian la interacción estudiante-estudiante, estudiante-grupo. Estos elementos fueron informados en investigaciones anteriores, como es el caso de Bravo (2004), quien coincide en que no hay correspondencia entre el método declarado por el profesor y el utilizado, lo que evidencia que los profesores no tienen clara la concepción metodológica seguida en su clase.

Esta situación se confirma también en la fase de control, porque si bien se sigue una concepción didáctica diversa, según las especificidades de las asignaturas, no son amplias las actividades reflexivas y autovalorativas que permitan una participación activa en el proceso. En escasas ocasiones se realizan actividades de control orientadas al análisis de las vías que pudieran ser posibles para solucionar un problema.

Estos datos se confirman al analizar los resultados de la figura 1. Nótese que es mayor el número de profesores que no favorece la comunicación interpersonal en la fase de orientación y evaluación y que es 
esta última en la que menos se identifica con las posibilidades de favorecer la comunicación interpersonal en el proceso de enseñanza-aprendizaje.

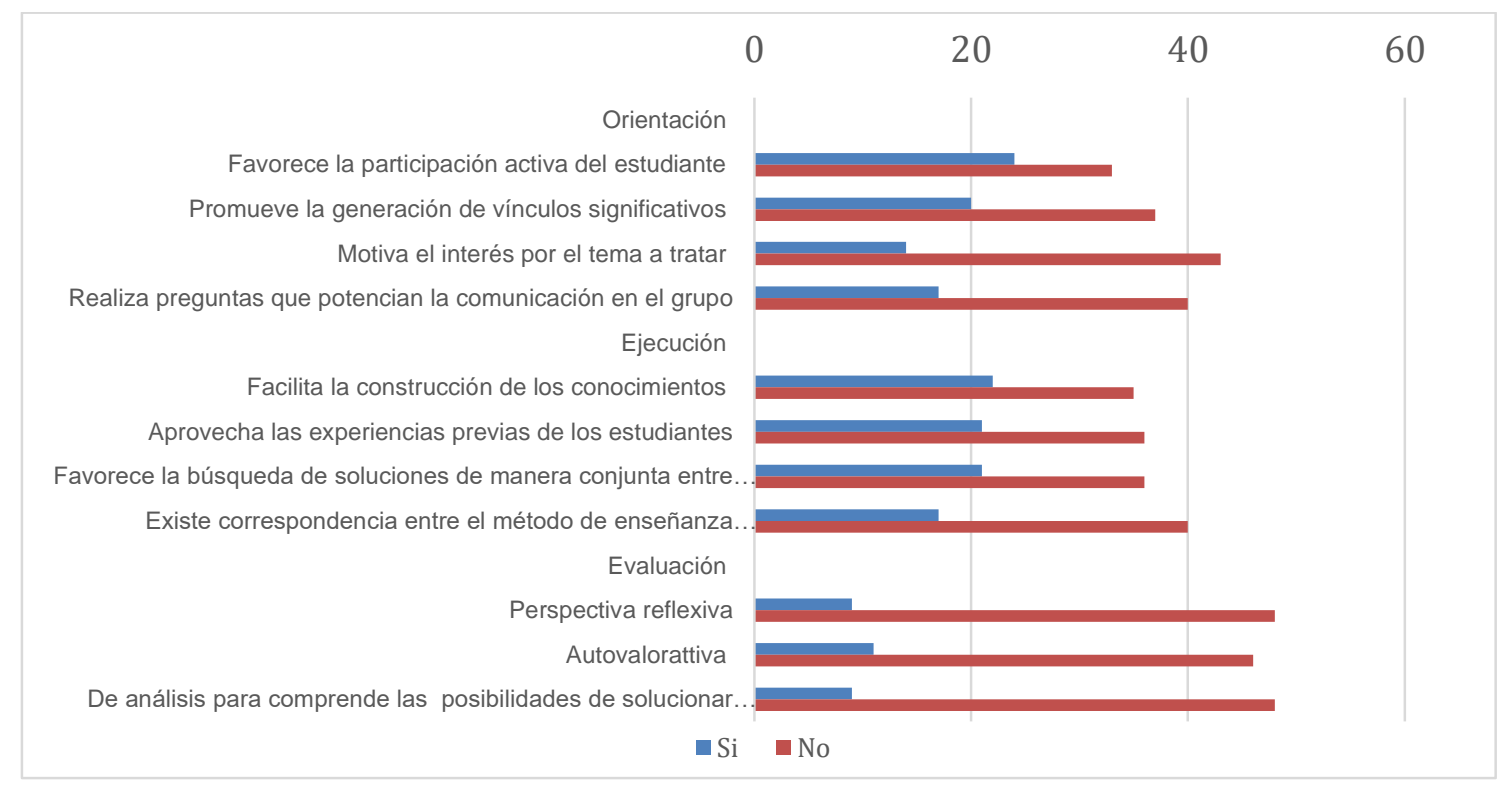

Figura 1. Representación de los resultados de la observación del proceso enseñanza- aprendizaje

Sin embargo, al sintetizar la frecuencia con que se manifiestan las interacciones entre profesor, estudiante y entre estudiantes (ver figura 2), es evidente que predomina el papel del profesor como principal protagonista del proceso, como lo sugieren los resultados de Amayuela (2005) en un contexto formativo universitario similar.

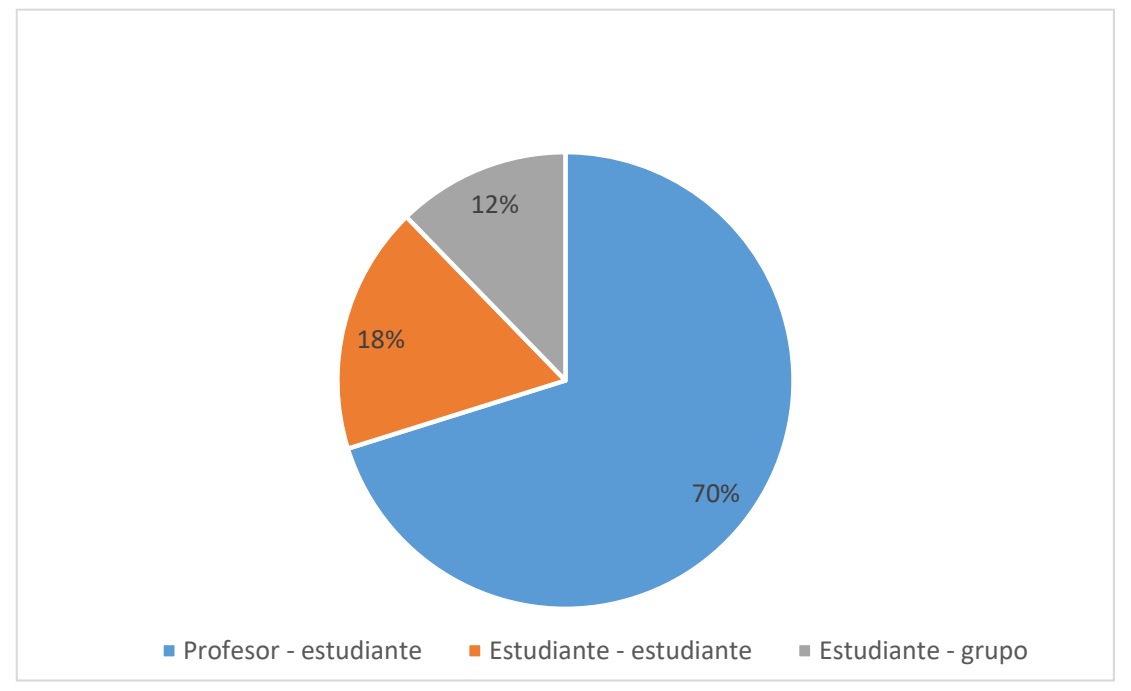

Figura 2. Representación del tipo de interacción que se establece en el proceso de enseñanza-aprendizaje

En resumen, la información recopilada en las observaciones reveló que las actividades realizadas en el proceso de enseñanza-aprendizaje no logran propiciar la comunicación interpersonal como es deseado, ya que los profesores se ven limitados a utilizar métodos o recursos que lo permitan. Además, es insuficiente la orientación (por parte de los profesores) de los aspectos que posibiliten que el estudiante pueda generar ideas, motivarse e indagar, aun cuando en varias actividades el profesor utilizaba múltiples interrogantes 
con el propósito de lograr la participación de los estudiantes.

En este sentido, en correspondencia con Bravo (2004), Amayuela (2005) y García (2015), las observaciones muestran que en general no se utilizan métodos que estimulen el diálogo, el pensamiento analítico o reflexivo, y es limitado el trabajo en grupos, aun cuando se indican tareas evaluativas por grupos.

En el registro de apuntes y comentarios asumidos como parte de la sistematización realizada por los investigadores se puede identificar que con frecuencia los profesores no conceden a los estudiantes un tiempo para que razonen y puedan construir conocimientos y expresar sus puntos de vista, como alertaban Jurado, Bravo y Avello (2017).

Se hizo evidente que con frecuencia se interrumpen las respuestas, se indica que no se está de acuerdo con lo que los alumnos dicen y se les pide que sinteticen o sean concretos, limitando la expresión oral. No se utilizan análisis de casos u otros métodos que posibiliten el debate, la reflexión y la toma de posición. Por el contrario, se estimula y valora como positivas las respuestas directas y reproductivas, lo cual limita la comunicación interpersonal en el proceso de enseñanza-aprendizaje.

\subsection{Autorreporte tipo inventario}

Al procesar los datos del autorreporte tipo inventario aplicado a 62 profesores de las carreras de Educación se confirma la idea de que los problemas detectados en las observaciones en clases están formando parte de las concepciones y prácticas de los profesores. El análisis de los datos permitió a los autores de esta investigación exponer los principales resultados:

Es de señalar que 47 participantes (75.80\%) manifiestan estar satisfechos con la forma en que se comunican con los estudiantes; aunque 59 profesores (95.16\%) quisieran poder tener otra forma de comunicarse con los mismos. Lo cual, como también manifiestan profesores participantes en investigaciones anteriores en otros contextos (Bravo, 2004; García, 2015; Tejeda, 2010), expresa insatisfacciones en la comunicación que actualmente se establece en el proceso de enseñanza-aprendizaje.

De igual forma, 41 profesores (66.12\%) señalan que les resulta difícil comunicarse con los alumnos en otros temas fuera de clase y 51 (82.25\%) de ellos expresan que existen momentos en que los estudiantes se desmotivan en sus clases y la comunicación no fluye adecuadamente, esto se corroboró en las observaciones en clases, en las cuales el profesor motiva al comenzar el tema a tratar, pero no mantiene la motivación en su desarrollo ni utiliza métodos que promuevan una participación activa.

Es significativo que 56 profesores (90.32\%) aseguren que si pudieran modificarían la forma en que habitualmente se comunican con los estudiantes; sin embargo, cuando tienen la posibilidad en la clase u otros espacios, no lo hacen. Cabe destacar que un gran número de profesores, 58 (93.54\%) no siempre usa los métodos adecuados a las características del grupo, de manera que se haga más asequible la exposición; a esta situación se agrega que a 58 (93.54\%) les resulta difícil desarrollar las habilidades comunicativas en sus estudiantes. Estos resultados contrastan con los hallazgos de Bravo (2004) y Portela (2015), quienes también sugieren la necesidad, a la luz de los resultados, de formar y orientar a los profesores en estos temas.

También se pudo constatar que 59 profesores (95.16\%) afirman que no logran despertar intereses cognoscitivos en los estudiantes y 54 (87.09\%) no logran que los estudiantes sientan la necesidad de comunicarse sistemáticamente con los profesores Esto puede deberse a que no atiendan sus características y necesidades emocionales y no se apoyan en las motivaciones de los mismos para impartir los nuevos contenidos, lo que limita que se cumpla el principio de la unidad entre lo afectivo y cognitivo en el proceso de enseñanza-aprendizaje. Además, 53 profesores (85.48\%) expresaron que su estado de ánimo no siempre es el más adecuado y sienten que afecta en la comunicación, lo cual puede deberse a las distintas actividades que debe cumplir en su rol de profesor. 


\subsection{Cuestionario a profesores}

De acuerdo con los datos que se extraen al procesar la información del cuestionario y que se representa en la figura 3, se infiere que los profesores reconocen que existen deficiencias en la manera en que el proceso de enseñanza-aprendizaje favorece la comunicación interpersonal; reconocen que hay fallas en la estructuración didáctica, advierten la implicación que tiene el método en esta situación y que si se introdujeran cambios en ésta podrían facilitar la comunicación interpersonal. De igual forma, se constató una alta disposición para el cambio en los métodos de enseñanza-aprendizaje, lo que se identifica como un aspecto positivo para llevar a cabo esta investigación.

En este orden, al explorar cómo el profesor contribuye a la comunicación interpersonal en el proceso enseñanza-aprendizaje, a través de un cuestionario de opinión (figura 3) se evidenció que en el ítem referido a si ejecuta tareas en equipo para favorecer la comunicación interpersonal, 45 profesores (72.58\%) indicaron que no, señalando en la justificación que les lleva mucho tiempo y deben cumplir con un programa; de igual manera, es significativo señalar que 50 profesores (80.64\%) consideraron que favorecen la comunicación y en la justificación precisaron que realizan preguntas para que los estudiantes respondan, lo que se corroboró en la observación a las actividades de los profesores y está en correspondencia con hallazgos anteriores (Tejeda, 2010), ya que muchas de las interrogantes realizadas en clases estaban orientadas a responder con monosílabos o no llevaban un análisis reflexivo.

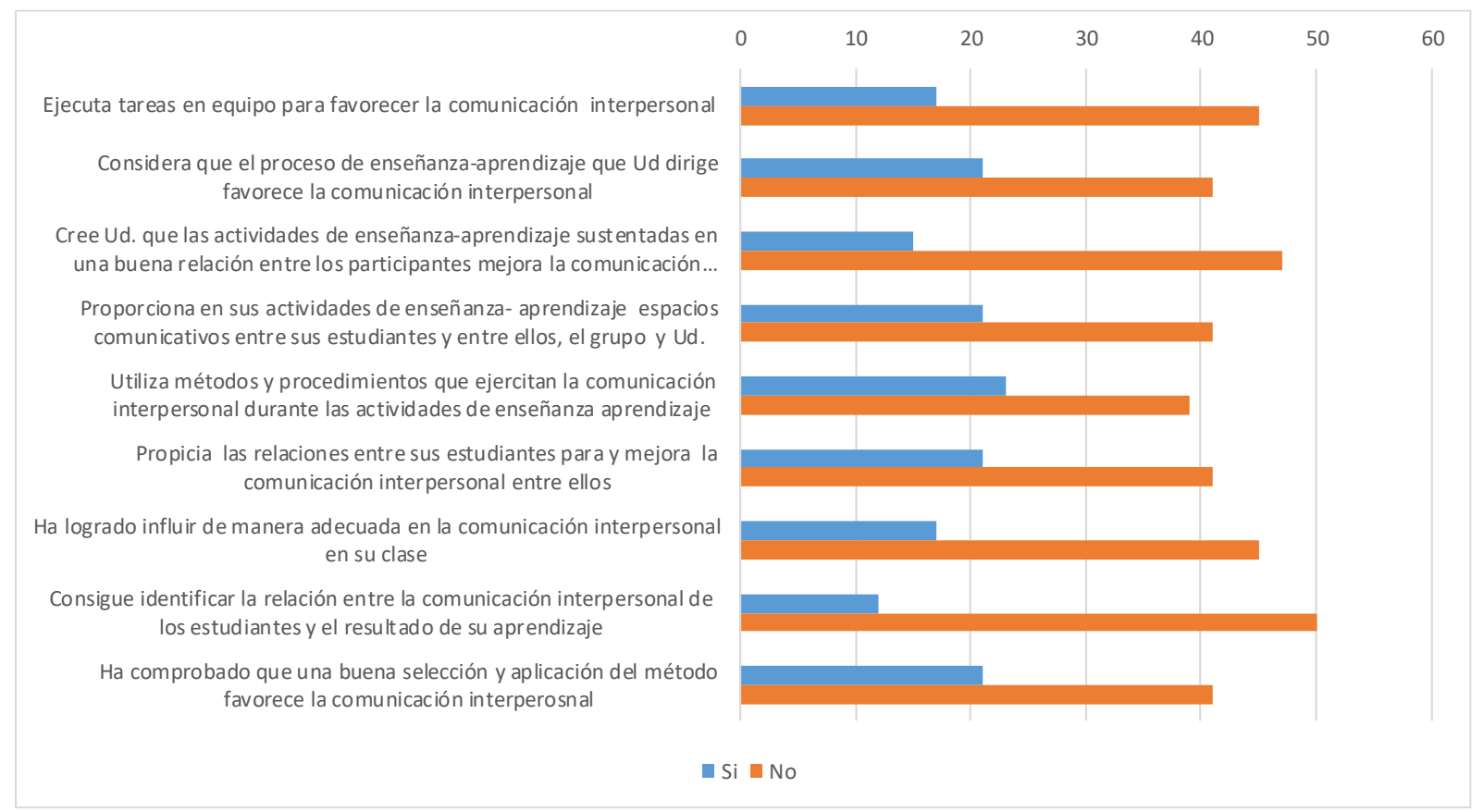

Figura 3. Representación de las concepciones de los profesores acerca de la comunicación en el proceso de enseñanza-aprendizaje

Un número significativo de profesores, 47 (75.80\%), no reconocen la importancia que tienen los intercambios comunicativos en el proceso de enseñanza-aprendizaje para mejorar la comunicación interpersonal en el grupo; sin embargo 39 (62.90\%) de ellos dijeron utilizar métodos que pueden estimular la comunicación, 40 (64.51\%) afirman que las relaciones entre sus estudiantes mejoran la comunicación interpersonal. Es posible identificar que la principal limitación que poseen los profesores es que no identifican la relación entre la comunicación interpersonal y los resultados de aprendizaje de los estudiantes, y esto se confirma cuando 50 (80.6\%) responden de manera negativa. 


\subsection{Cuestionario a estudiantes}

Al contrastar estos criterios con el cuestionario de opinión que se aplicó a los estudiantes en la Licenciatura en Educación se pudo confirmar que se ratifican las dificultades presentes en el proceso de enseñanzaaprendizaje para la comunicación interpersonal: más del 60 \% de los estudiantes marcó, con cero o con signo de interrogación, el ítem del cuestionario correspondiente a los métodos utilizados por el profesor, lo cual revela las insuficiencias en la percepción de sus necesidades, la utilización de recursos para despertar motivaciones e intereses o influir en su modo de actuar y crear las condiciones para que se realice la comunicación interpersonal, estudiante-estudiante, estudiante-grupo, durante las actividades de enseñanza-aprendizaje orientadas.

Se aprecia así una alta crítica a las clases, pues éstas no aprovechan las oportunidades para estimular la expresión de sentimientos, emociones, hacerlos sentir confiados y apoyados y destacan al profesor como el principal responsable de estos procesos.

Al aplicar un segundo cuestionario a los mismos estudiantes orientado a revelar la valoración que poseen acerca de la comunicación interpersonal y los aprendizajes que han logrado a través de la misma, se evidencian las acciones que con mayor frecuencia identifican al proceso de enseñanza-aprendizaje. En particular, los mayores porcentajes confirman la utilización de métodos basados en preguntas y respuestas con 300 estudiantes para 84.51\%, la expresión de ideas con 298 para 83.94\%; la explicación con 350 que representa 98.59\%; la descripción con 326 para 91\%; el análisis de problemas con 346 para un 97.46\%; y la aplicación de conocimientos basada en algoritmo con 354 para $99.71 \%$.

Lo anterior evidencia que estos métodos no son suficientes para favorecer la comunicación interpersonal; las actividades se orientan más al trabajo grupal, pero no se aplican técnicas o dinámicas grupales que favorezcan la expresión cognitiva y valorativa acerca del contenido de la enseñanza y es muy regulada la red comunicativa, porque predomina la unidireccionalidad profesor-estudiantes, en la cual el primero interrumpe con frecuencia la comunicación entre estudiantes. Es evidente que los profesores, como sugieren otros autores (García, 2015; Turró, 2017), en muchas ocasiones no aprovechan la comunicación interpersonal que se da de manera informal dentro del proceso de enseñanza-aprendizaje.

\subsection{Segundo cuestionario a estudiantes}

Al analizar los resultados del segundo cuestionario a los estudiantes resaltan -entre las las acciones que más frecuentemente identifican en el proceso de enseñanza-aprendizaje- preguntas, vivencias e interacciones. Dentro de la información que brinda el profesor, utiliza métodos basados en la descripción, la explicación y el trabajo con libros de texto u otros materiales. Por último, durante el tratamiento del contenido desarrollan actividades orientadas mayoritariamente al análisis de problemas y situaciones prácticas reales o simuladas, al análisis crítico y la argumentación científica, al análisis de problemáticas en condiciones similares, reflexivas y autovalorativas.

De igual modo, al caracterizar la contribución del proceso de enseñanza-aprendizaje a la comunicación interpersonal, se evidencia una tendencia negativa hacia las habilidades de observación, expresión oral, escucha y expresión escrita, y lo mismo ocurrió con la contribución del lenguaje extraverbal, el manejo de la voz, el orden de las ideas, así como la ortografía y la lógica interna del mensaje, las cuales fueron mayormente poco valoradas.

Resulta interesante que los estudiantes identifican como logros positivos del proceso de enseñanzaaprendizaje disfrutar la comunicación con los demás, la tolerancia a ideas incongruentes. Ponderan como negativo con altos porcentajes (96\%) que no aprenden a explicitar los sentimientos en el momento oportuno y despertar emociones en los demás; ciertamente los estudiantes no han logrado desarrollar los aspectos esenciales de una relación interpersonal adecuada. 


\section{Conclusiones}

Al contrastar la información que aportaron los instrumentos aplicados se concluye que si bien los profesores reconocen el papel de la comunicación interpersonal en el proceso de enseñanza-aprendizaje no poseen una concepción metodológica que favorezca la comunicación interpersonal, pues mantienen una metodología tradicional, unilateral y desaprovechan las posibilidades del intercambio.

En general se aprecia, por un lado, una limitación en la proyección didáctica de la comunicación interpersonal en el proceso de enseñanza-aprendizaje, lo cual se evidencia en la falta de diálogo, carencia de actividades grupales durante las clases, insuficiencia en la personalización del proceso, afectando la independencia. Por otra parte, no se propicia un clima afectivo que permita crear situaciones de aprendizaje, facilitadora de una realización abierta en la comunicación de conocimientos, valoraciones, sentimiento y nuevas ideas, basadas en las experiencias personales y grupales. Estos resultados están en correspondencia con investigaciones precedentes como en Corujeira (2014) y García (2015).

Lo apuntado justifica la necesidad de esta investigación, identificando como potencial la disposición y apoyo de directivos y profesores para llevarla a cabo al expresar durante su transcurso el interés por el tema y confirmar de manera crítica los resultados que se obtuvieron, lo cual quedó manifiesto en el interés de que los investigadores procedieran a la elaboración de una propuesta que permita la orientación y formación de los docentes con respecto a la necesidad de una adecuada comunicación interpersonal en el proceso de enseñanza-aprendizaje.

Para responder a esta exigencia los autores confrontaron las ideas teóricas y los resultados de la práctica, así como resultados alcanzados en investigaciones precedentes (Bravo, 2004; García, 2015; Tejeda, 2010), asumiendo que mediante este proceso se podían generar las decisiones básicas para la elaboración de la propuesta. En este sentido, fue necesario desplegar un grupo de reflexiones individuales que fueron compartidas con profesores y directivos implicados en este proyecto.

A la luz de los resultados y la revisión de las concepciones teóricas, se decidió presentar el resultado como un sistema de métodos (Jurado et al., 2017). En el cual se consideró que en el proceso de enseñanzaaprendizaje, en tanto secuencia de actividades estructuradas e intencionadas, confluyen y se integran varios métodos con un carácter jerárquico y de complementación didáctica. Por esta razón, el enfoque sistémico que caracteriza al proceso de enseñanza-aprendizaje se convierte en condición que asumen los métodos al ser utilizados.

\section{Referencias}

Alpizar, L. B. (2017). La comunicación pedagógica en los docentes del nuevo plan de formación en Medicina Integral Comunitaria. Educación Médica Superior, 31(2).

http://www.ems.sld.cu/index.php/ems/article/view/946

Amayuela, M. C. (2005). Estrategia didáctica para la transposición de la cultura psicológica sobre la simetría funcional comunicativa al contexto formativo universitario (Tesis de Doctorado). Universidad de Camagüey, Cuba.

Berger, J. (2014). Word of mouth and interpersonal communication: a review and directions for future research. Journal of Consumer Psychology, 24(4), 586-607. https://doi.org/10.1016/i.jcps.2014.05.002

Bravo, G. (2004). Una estrategia para la formación permanente del profesorado de Secundaria Básica desde la Comunicación Educativa (Tesis de Doctorado). Universidad de Cienfuegos, Cuba.

Cohen, L., Manion, L. y Morrison, K. (2013). Research methods in education (7a. ed.). Oxon.

Contreras, J. (1990). La enseñanza como comunicación. Enseñanza, Currículum y profesorado. Akal. 
Corujeira, J. (2014). Comunicación interpersonal y metodologías de la innovación. Una experiencia heurística en el aula aplicando redes semánticas. Disertaciones, 7(2).

http://erevistas.saber.ula.ve/index.php/Disertaciones/article/view/4618

Elkonin, D. B. (1982). Acerca del problema de la periodización del desarrollo psíquico en la edad infantil. En Superación para profesores de Psicología (pp. 144-154). Pueblo y Educación.

García, M. G. (2015). Comunicación y relaciones interpersonales. Tendencias Pedagógicas, 2, 11-17.

Hargie, O. (2016). Skilled interpersonal communication: research, theory and practice. Routledge.

Heredero, E. S. y Garrido, P. (2017). Desarrollo de la inteligencia interpersonal e intrapersonal en educación primaria a partir del uso de tecnologías de información y comunicación: estudio de casos. Notandum 44-45. http://dx.doi.org/10.4025/notandum.44.15

Hernández, R., Fernández, C. y Baptista, P. (2014). Metodología de la investigación (6a. ed.). Mc Graw Hill.

Johnson, Z. D., LaBelle, S. y Waldeck, J. H. (2017). A cautious approach to reliance on interpersonal communication frameworks: the importance of context in instructional communication research. Communication Education, 66(1), 115-117

Jurado, M., Bravo, G., Avello, R. (2017). Propuesta de un sistema de métodos para la comunicación interpersonal en la carrera de Licenciatura en Educación. Medisur, 15(6).

http://medisur.sld.cu/index.php/medisur/article/view/3747

Jurado, M., Bravo, G. y Muñoz, I. (2016). La comunicación y los métodos de enseñanza aprendizaje en el licenciado en educación. Revista Sarance, 36, 141-151.

Leontiev, A. N. (1985). Actividad, conciencia y personalidad. Pueblo y Educación.

Lomov, F. B. (1987). El problema de la comunicación en psicología. Ciencias Sociales.

Portela, G. (2015). Los métodos y técnicas participativas en el proceso enseñanza aprendizaje. Mediciego, 11(1).

Punyanunt-Carter, N. M. y Arias, V. S. (2017). The interplay between interpersonal communication and instructional communication. Communication Education, 66(1), 118-120.

http://dx.doi.org/10.1080/03634523.2016.1221512

Sánchez, M. (2000). La conversa a l'aula. [La conversación en el aula]. Articles de Didàctica de la Llengua i la literatura, 21,109-118. http://articles.grao.com/revistes/articles/021-literatura-juvenil/la-conversa-a-l-aula

Sánchez, B., y Boronat, J. (2014). Coaching educativo: modelo para el desarrollo de competencias intra e interpersonales. Educación XX1, 17(1), 221-242.

http://revistas.uned.es/index.php/educacionXX1/article/view/10712

Tejeda J. (2010). Las habilidades comunicativas en los estudiantes del ciclo clínico de la carrera de Medicina (Tesis de Doctorado). Universidad de Cienfuegos, Cuba.

Turró, M. (2017). Las interacciones orales en pequeños grupos una oportunidad de aprendizaje cognitivo, social e identitario. Didacticae, 1, 111-129. https://doi.org/10.1344/did.2017.1.111-129 